\title{
Space-variant ambiguity function processor
}

\author{
Demetri Psaltis and David Casasent
}

\begin{abstract}
The ambiguity function that results from the space-variant optical processing of nonlinear coded waveforms is analyzed. The space-variant signal processor considered is realized by coordinate transformations. Specifically we consider $x=\ln t$, which results in a Mellin transform-based Doppler-invariant signal processor. Ambiguity function data are provided for a nonlinearly coded 13-bit Barker code.
\end{abstract}

\section{Introduction}

Space-variant optical signal processing ${ }^{1-7}$ has received considerable attention in recent years. The Mellin transform ${ }^{8}$ is a space-variant operation of particular interest in signal processing. It is realized by performing an optical Fourier transform on a coordinate transformed version of the input data using techniques originally suggested for use in image restoration and deblurring. ${ }^{9,10}$ As we showed earlier, ${ }^{1,2}$ these techniques can be used to produce a Doppler-invariant optical signal processor that avoids the need for a Doppler search or a Doppler filter bank. Since, this signal processor is a space-variant system, a time search rather than a scale search is needed unless a parallel and real-time coordinate transformation system is used. The use of computer generated holograms ${ }^{11-13}$ and astigmatic optical systems ${ }^{14,15}$ provides two methods by which the required coordinate transformation can be performed. The former system has space-bandwidth limitations, whereas the latter is of use only for introducing 1-D distortions onto 1-D functions and has a large light loss. However, refinements in such technologies and use of alternate approaches such as multiple holograms ${ }^{16}$ and phase-coded reference beams ${ }^{17}$ appear to make our initial Mellin transform Dopplerinvariant signal processor concept $\mathrm{t}^{1,2}$ more realizable in the near future. For this reason we now expand upon our earlier work in space-variant signal processing by considering the use of such systems with coded waveforms with emphasis on the resultant ambiguity function.

The authors are with Carnegie-Mellon University, Department of Electrical Engineering, Pittsburgh, Pennsylvania 15213.

Received 25 August 1978.

0003-6935/79/111869-06\$00.50/0.

(c) 1979 Optical Society of America.
As we noted earlier, ${ }^{18,19}$ the transmitted waveform for such space-variant signal processors must be a nonlinear waveform (produced by coordinate transforming presently used coded signals). We discuss this issue in Sec. III following a brief review (Sec. II) of our prior work on space-variant signal processing. Since the resultant systems are space-variant, conventional analytical tools are not useful, and a numerical analysis is required. To obtain more explicit results, we restrict specific discussion to the Mellin transform with its $x=$ $\ln t$ coordinate transformation and the 13-bit binary phase-coded Barker waveform. No loss of generality in the over-all concept of nonlinear waveforms and space-variant processing using coordinate transformations results by concentrating on this specific scenario.

In Secs. IV and V, we derive the ambiguity function for such a system and discuss the unique waveform and system design features afforded by this novel approach to signal processing. The resultant transmitted signals are a new class of nonlinear spread spectrum code that promises to open new research areas for waveform and system design and communications.

\section{Space-Variant Signal Processing}

We restrict attention to space-variant signal processing and specifically to the realization of such systems by the use of coordinate transformations (as originally suggested for image enhancement ${ }^{9,10}$ ). We also consider only the optical Mellin transform because of its unique features, and we consider the Dopplershifted version $f^{\prime}(t)=f(a t)$ of $f(t)$. If we apply the coordinate tranformation $t=\exp x$ to these signals we obtain $f_{1}(x)=f(\exp x)$ and $f_{1}^{\prime}(x)=f(a \exp x)$. If we now form their correlation we find ${ }^{1,2}$

$$
R(\hat{x})=f_{1}^{\prime}(x) \circledast f_{1}(x)=f(\exp x) \circledast f(\exp x) * \delta(\hat{x}-\ln a),
$$

where $x$ and $\hat{x}$ are used to denote the input and output plane variables, respectively. 
Thus, as we demonstrated earlier, the correlation of two properly coordinate-transformed signals is Doppler-invariant, and the Doppler parameter $a$ can be found from the location of the resultant correlation peak. Furthermore, only a single-channel 1-D optical correlation is required. Equation (1) can be realized by forming the Fourier transform (symbolized by the operator $\mathscr{7}$ ) of the product of the Fourier transforms $F_{1}^{*}(u)$ and $F_{1}^{\prime}(u)$ of $f_{1}(x)$ and $f_{1}^{*}(x)$. Since the Fourier transform of $f(\exp x)$ is the Mellin transform $M(u)$ of $f(t)$, we can rewrite Eq. (1) as

$$
R(\hat{x})=f_{1}^{\prime}(x) \circledast f_{1}(x)=\mathcal{F}\left[F_{1}^{\prime}(u) F_{1}^{*}(u)\right]=\mathscr{F}\left[M^{\prime}(u) M^{*}(u)\right],
$$

and thus we refer to such a processor as a Mellin transform correlator. The system by which $M(u)$ is produced from $f(t)$ is a space-variant processor because of the coordinate-transformation preprocessing step required.

\section{Nonlinear Waveforms}

If $f(x)$ is a phase coded waveform, we can write it as

$$
f(x)=\cos \left[\omega_{0} x+\phi(x)\right]=a(x) \cos \left(\omega_{0} x\right),
$$

where for a 13-bit biphase Barker code ${ }^{20}$

$$
\begin{aligned}
a_{n}(x) & =\exp \left[j \phi_{n}(x)\right] \\
& =+1,+1,+1,+1,+1,-1,-1,+1,+1,-1,+1,-1,+1 .
\end{aligned}
$$

Letting $x=\exp (t)$ in Eqs. (3), we obtain

$$
f_{1}(t)=\cos \left[\omega_{0} e^{t}+\phi\left(e^{t}\right)\right]=f\left(e^{t}\right) .
$$

We would normally form the correlation of $f_{1}(t)$ and a coordinate-transformed distorted (Doppler-shifted and/or range delayed) version $f_{1}^{\prime}(t)$ of $f_{1}(t)$.

As we recently noted, ${ }^{18,19}$ the nonlinear carrier in Eq. (4) dominates the characteristics of the correlation and ambiguity function; in addition waveform design flexibility is not present. Of more concern is the fact that when a coordinate-transformed version of the reference and input waveforms are correlated, we are in effect correlating on the coordinate transformation rather than the coded waveform. For these reasons, a pure uncoded sinusoidal signal will correlate nearly as well as the original waveform, since both are subjected to the same coordinate transformation. Such a signal corresponds to a discrete jammer in a radar application.

In Fig. 1, we show an isometric (pseudo-3-D) display of the optically produced output autocorrelation of Eq. (4) for a nonlinear (coordinate transformed) 13-bit Barker code as described by Eq. (3) with a spatial $B W$ $=11.4$ cycles $/ \mathrm{mm}$. The coordinate transformation tapers and spreads the spectrum of the signal, and the narrow correlation peak shown in Fig. 1 results. However, as we showed earlier, ${ }^{18,19}$ better resolution would result if a linearly coded waveform of the same bandwidth were used.

For the reasons noted above, the optimum coded waveform to transmit is one which, when coordinate transformed, results in a normal coded waveform, with the good ambiguity function properties and flexible waveform design features afforded by the use of coded

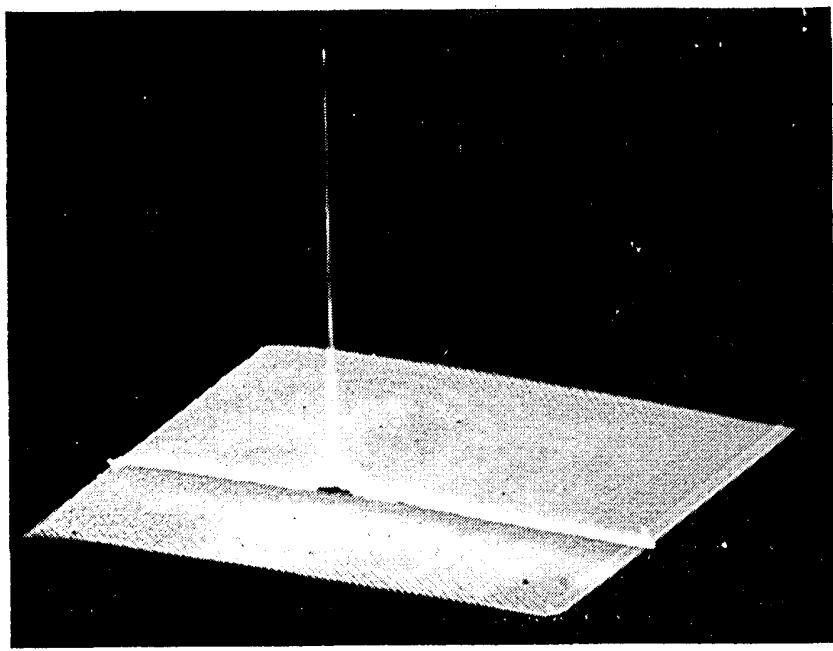

Fig. 1. Isometric (pseudo-3-D) display of the optical correlation in Eq. (2).

waveforms. Thus the optimum use of coded waveforms in the type of space-variant optical processor we consider is achieved by applying the coordinate transformation to the coded waveform prior to transmission, applying an inverse coordinate transformation to the received signal and then correlating this signal with the original linear coded waveform.

Thus, for a Doppler invariant Mellin transform signal processor using coded waveforms, we apply the coordinate transformation

$$
x=\ln \left(t+t_{0}\right) \text { for } 0<t<T
$$

to Eq. (3a) [where $T$ is the duration of $f(t)]$ and transmit the nonlinear coded waveform

$$
f_{t}(t)=\cos \left(\omega_{0} \ln \left(t+t_{0}\right)+\phi\left[\ln \left(t+t_{0}\right)\right]\right\}
$$

In Eqs. (5) and (6), $t_{0}>0$ is a constant offset in the time reference that allows us to avoid the difficulty associated with implementing the log of zero. In practice, we will receive a Doppler shifted and/or range delayed version $f_{r}(t)$ of Eq. (6), and we apply to it the inverse transformation

$$
t=\exp x-\hat{t},
$$

where $\hat{t}$ is a delay variable. The delay variable $\hat{t}$ is included in Eq. (7) to indicate that the coordinate transformation is performed in real time. This yields a new function

$$
f_{1}(\hat{t}, x)=f_{t}\left(e^{x}-\hat{t}\right),
$$

which is then correlated with the reference waveform $f(x)$ in Eq. (3a). The block diagram for a space-variant optical processor using coordinate transformations is shown in Fig. 2.

The processing required to generate the ambiguity function $\chi(\hat{t}, \hat{x})=\chi(\tau, \nu)$ is described by

$$
\begin{aligned}
\chi(\hat{t}, \hat{x})= & f_{1}(\hat{t}, x) \circledast f_{t}(x)=\int f_{1}\left(e^{x}-\hat{t}\right) f_{t}(x-\hat{x}) d x \\
= & \iint_{0} \cos \left\{\omega_{0} \ln \left(e^{x}+t_{0}-\hat{t}\right)+\phi\left[\ln \left(e^{x}+t_{0}-\hat{t}\right)\right]\right\} \\
& \times \cos \left[\omega_{0}(x+\hat{x})+\phi(x+\hat{x})\right] d x,
\end{aligned}
$$




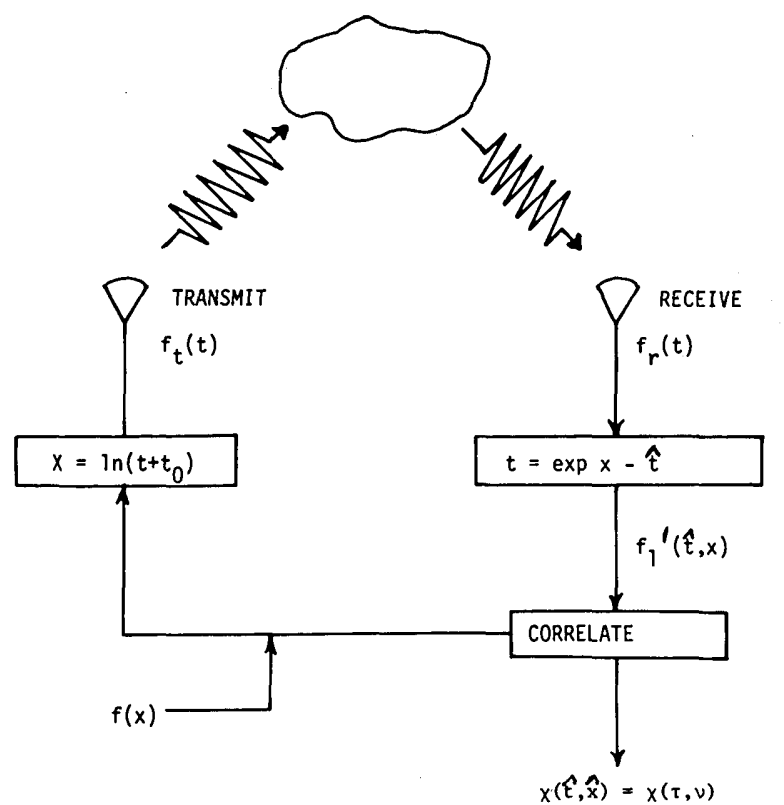

Fig. 2. Block diagram of a space-variant processor for coded waveforms using coordinate transformations.

where $\hat{x}$ is the correlation variable. The above equation can be shown to be equivalent to the wideband ambiguity function.

Doppler shifts between $f_{t}(t)$ and $f_{r}(t)$ are converted to shifts in the location $x$ of the received signal by the coordinate transformation described by Eq. (7). Thus, the output correlation variable $\hat{x}$ now corresponds to Doppler. Because of the coordinate transformation applied to the received signal $f_{r}(t)$, this is a Mellin transform type correlator. Because the Mellin transform is a space-variant operation, a time search rather than a Doppler search is necessary. This is included in the form of the coordinate transformation given in Eq. (7), which must be performed continuously for all time $t$. ( $t$ is input signal time, and $x$ is distance across $P_{0}$.) By properly utilizing the time and space variables of an optical processor and by use of the real-time and fully parallel space-variant coordinate transformation optical systems noted in Sec. I, such space-variant optical signal processors appear feasible. Our present concern is with an analysis of the.resultant ambiguity surface and the waveform and system design aspects of the systems in Figs. 2 and 3.

An optical system that can realize these required operations is shown in Fig. 3. In practice, $L_{1}$ and $L_{2}$ would be a cylindrical/spherical lens pair that performs the Fourier transform horizontally while imaging vertically. For simplicity, only the spherical lens portion of the system is shown in Fig. 3. In this single-channel 1 -D system, the vertical $\hat{x}$ location of the output in $P_{2}$ denotes Doppler $\nu$, whereas the time $\hat{t}$ at which the output occurs corresponds to range $\tau$. Thus, an output ambiguity surface ${ }^{21}$ is obtainable on a single-channel $1-D$ optical system by proper use of the time and space variables at our disposal.
In this system, the Doppler axis is formed by a Mellin transform correlation in space, and the $\tau$ range search is achieved by a time search. These are some of the unique features of this space variant processor. In more conventional multiplexed space invariant processors, the correlation is usually performed in time, and the Doppler shifts are always introduced in space. Because the resultant ambiguity surface obtained in our proposed space-variant processor differs from the conventional one, we will consider the characteristics of the resultant output surface in detail in Sec. IV.

\section{Ambiguity Function}

The purpose of any ambiguity function is threefold. First, it must provide a discrimination ability; this is usually obtained by a single dominant peak in the ambiguity surface. The peak value is used to determine whether the particular signal is present. The location of this central peak in the 2-D output ambiguity function space provides two more vital pieces of information, Doppler and range. The ambiguity function produced by the space-variant processor described in the previous section must provide the same information if it is to be used in place of the conventional one. We will demonstrate that indeed it does provide discrimination ability, as well as Doppler and range information, and will derive the parameters that determine its performance.

We first verify that Eq. (9) attains a maximum and determine where it occurs. Application of the Schwartz inequality is not allowable since the energy in the coordinate transformed signal changes with time. At $\hat{t}=t_{0}$, Eq. (9) reduces to the autocorrelation of Eq. (3) which peaks at $\hat{x}=0$ along the $\hat{x}$ direction. To show that $\chi(\hat{t}, \hat{x})$ also peaks at $\hat{t}=t_{0}$ along the $\hat{t}$ direction, we use the method of stationary phase. ${ }^{22}$ This involves the approximation that the major contribution to an integral rises from the points at which the derivative of its total phase function is zero. For an integral of the form

$$
A=\int f(x) \exp [j \phi(x)] d x,
$$

the major contribution occurs at the points $x_{0}$ where

$$
\left.\frac{\partial \phi(x)}{\partial x}\right|_{x=x_{0}}=0 .
$$

The contribution from these points is

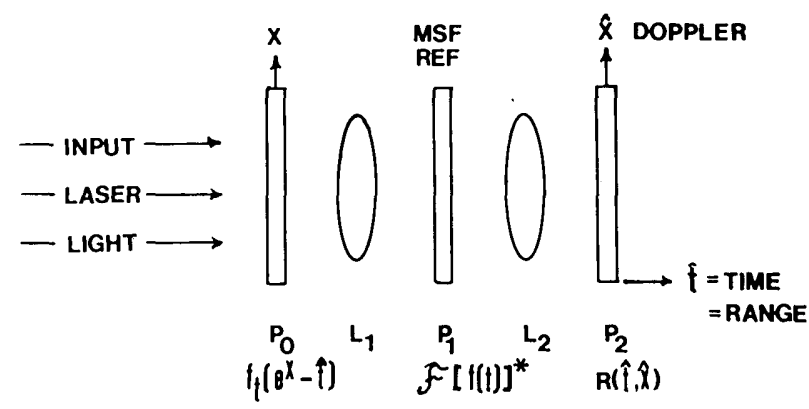

Fig. 3. Schematic diagram of a space-variant optical signal processor for coded waveforms using coordinate transformations. 


$$
|A|^{2}=\frac{\left|f\left(x_{0}\right)\right|^{2}}{\partial^{2} \phi /\left.\partial x^{2}\right|_{x=x_{0}}},
$$

where $x_{0}$ is the point that solves Eq. (11). Applying Eq. (11) to Eq. (9) we find

$$
\frac{e^{x}}{e^{x}+t_{0}-\hat{t}}\left[\omega_{0}+\frac{\partial \phi(x)}{\partial x}\right]=\omega_{0}+\frac{\partial \phi(x+\hat{x})}{\partial x},
$$

which is satisfied for $\hat{t}=t_{0}$ and $\hat{x}=0$. Although other sets of $x, \hat{x}$ and $\hat{t}$ values satisfy Eq. (13), only $\hat{t}=t_{0}$ and $\hat{x}=0$ satisfy Eq. (13) for all $x$. At the point $(\hat{x}=0, \hat{t}=$ $t_{0}$ ), the contribution to Eq. (9) occurs along the entire $x$ line (rather than at only one point or only over a small $\Delta x$ region of $P_{Q}$ ), and thus Eq. (9) will have a much higher value at $\hat{t}=t_{0}$ and $\hat{x}=0$ than at any other $\hat{x}$ and $\hat{t}$ values. This same condition can be realized using Eq. (12) and observing that it becomes infinite only at $\hat{x}=$ 0 and $\hat{t}=t_{0}$.

We next consider the effects of a range delay $\tau$ in time in the received signal. For this case, Eq. (6) becomes

$$
f_{r}(t-\tau)=\cos \left\{\omega_{0} \ln \left(t-\tau+t_{0}\right)+\phi\left[\ln \left(t-\tau+t_{0}\right)\right]\right\} .
$$

When the coordinate transformation in Eq. (8) is applied to Eq. (14), we replace $\hat{t}$ by $\hat{t}-\tau$ in Eq. (9). Clearly the resultant ambiguity function now peaks at $\hat{t}=t_{0}+\tau$ rather than at $\hat{t}=t_{0}$ (as occurred for the case of no range delay). Of more concern is that this is simply a shift of $\chi(\hat{t}, \hat{x})$ in the $\hat{t}$ output axis with no change in the shape of the function and with the $\hat{x}$ axes unaffected.

We finally consider the effects of a Doppler shift in the received signal. We describe this as a time scaling $t \rightarrow a t$ in the received signal. (Such a formulation is applicable for both the narrowband and wideband functions.) For this case, Eq. (6) becomes

$$
f_{r}(a t)=\cos \left\{\omega_{0} \ln \left(a t+t_{0}\right)+\phi\left[\ln \left(a t+t_{0}\right)\right]\right\} .
$$

When Eq. (8) is applied to Eq. (15), we obtain

$$
\begin{aligned}
f\left(a e^{x}-a \hat{t}\right)= & \cos \left(\omega_{0} \ln \left[\exp (x+\ln a)-a \hat{t}+t_{0}\right]\right. \\
& \left.+\phi\left(\ln \left[\exp (x+\ln a)-a \hat{t}+t_{0}\right]\right\}\right) .
\end{aligned}
$$

From Eq. (16) we see that the Doppler scale change has been transformed into a shift in $x$ by $\ln a$. The resultant ambiguity function is thus shifted in $\hat{x}$ by $\ln a$ with the location of the peak in $\hat{t}$ changed from $\hat{t}=t_{0}$ to $\hat{t}=$ $t_{0} / a$.

When a Doppler shift occurs, the cross-coupling of the estimates of range and Doppler results because time and space are coupled by the coordinate transformation of Eq. (7). When range $\tau$ and Doppler shifts $\nu$ are simultaneously present, we can find $\nu$ from the location $\hat{x}=\ln a$ of the $\chi(\hat{t}, \hat{x})$ peak in $\hat{x}$ and thus unambiguously determine $\tau$ and $\nu$. The use of the Mellin transform correlation and the space-integrating moving window correlator of Fig. 3 allows us to realize the 2-D ambiguity surface on a single channel optical processor. Because $\tau$ and $\nu$ are coupled when $\nu$ is present, less $\Delta \hat{t}$ resolution results when noise is present because an estimate of $\nu$ is used to provide an estimate of $\tau$. The superior resolution $\Delta \hat{t}$ of the system of Fig. 3 (see Sec. V) and the system's ability to reject noise and interference (the subject of a future paper) offset this loss, however.

\section{Waveform Design}

We now consider how the waveform parameters, the coordinate transformation chosen, and the parameters of the components in the processor of Fig. 3 affect the system's performance and the characteristics of the resultant ambiguity function. We denote the carrier, bandwidth, and pulse length of the original linear Barker code of Eq. (3) by $\omega_{0}, B W$, and $T$. The same parameters for the nonlinear Barker-coded transmitted signal of Eq. (6) are $\omega_{0}, B W_{t}$, and $T_{t}$. For the coordinate transformed version of $f_{t}$ in Eq. (8) we obtain $\omega_{0}$, $B W_{C T}=B W$, and $T_{C T}=T$.

Since different scenarios impose different conditions and requirements, there is no one set of system parameters to optimize. Thus, we first establish a general set of parameter relationships for various performance goals. The two major design factors at our disposal are the choice of the waveform used and the coordinate transformation. In our case, the form of the coordinate transformation is fixed, but the portion of coordinate transformation space used can be varied as well as $\omega_{0}$, $B W, T$, and $t_{0}$ for the waveform.

The phase function $\phi(x)$ determines $B W$ and hence the system's Doppler resolution $\Delta \hat{t}$. We now show that the phase code $\phi(x)$ chosen does not in general affect the bandwidth $B W_{t}$ of the transmitted waveform $f_{t}(t)$.

To estimate the spectrum of Eq. (6), we differentiate the argument in Eq. (6) and find the instantaneous frequency to be

$$
f_{i}(t)=\left(\omega_{0}+\partial \phi / \partial y\right) /\left(t+t_{0}\right)
$$

for $0<t<T_{t}$, where $y=\ln \left(t+t_{0}\right)$. From Eq. (17), we find

$$
B W_{t} \simeq\left(\omega_{0} T_{t}\right) /\left[\left(t_{0}+T_{t}\right) t_{0}\right],
$$

where the approximation in (18) is valid if $\partial \phi / \partial y \ll \omega_{0}$ for $t=0$ and $t=T_{t}$. This is easily satisfied for large $\omega_{0}$ or when $\phi\left[\ln \left(t_{0}+t\right)\right]$ is slowly varying around $t=0$ and $t=T_{t}$. When $\phi(x)$ is chosen as indicated above, $B W_{t}$ is essentially independent of $\phi(x)$. Since $\phi(x)$ determines $B W$, which in turn determines the system's Doppler resolution $\Delta \hat{x}, B W$ and $\Delta \hat{x}$ are independent of $B W_{t}$.

Let us now consider the range accuracy $\Delta \hat{t}$ of this system and what dominates it. Since the carrier at $P_{0}$ is nonconstant for $\hat{t} \neq t_{0}$, the nonlinear carrier dominates Eq. (9) and the shape in $\hat{t}$ of the $\chi$ surface around the peak. This occurs because the carrier of the signal at $P_{0}$ matches that of Eq. (3) at only one $x^{\prime}$ point or only over some $\Delta x^{\prime}$ portion of $P_{0}$. The larger $B W_{t}$ is, the faster (in time $\hat{t}$ ) the signal at $P_{0}$ departs from the signal at $P_{1}$. Thus the higher the frequency change within a given time interval of $f_{t}$, the better $\Delta \hat{t}$ becomes. The signal in Fig. 3 decorrelates in $\hat{t}$ because of the nonconstant carrier for much the same reasons that decorrelation occurs in Doppler as the carrier changes in the conventional system. As $\omega_{0}$ increases, $B W_{t}$ increases, and hence $\Delta \hat{x}$ improves.

From these remarks, we see that $\phi(x)$ determines $B W$ but does not appreciably affect $B W_{t}$, which is dominated by the nonlinear carrier. Since $B W_{t}$ determines 


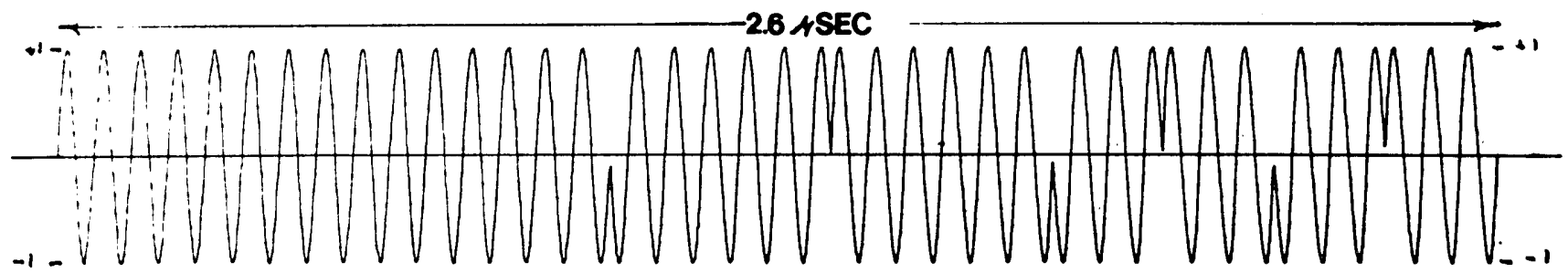

(a)

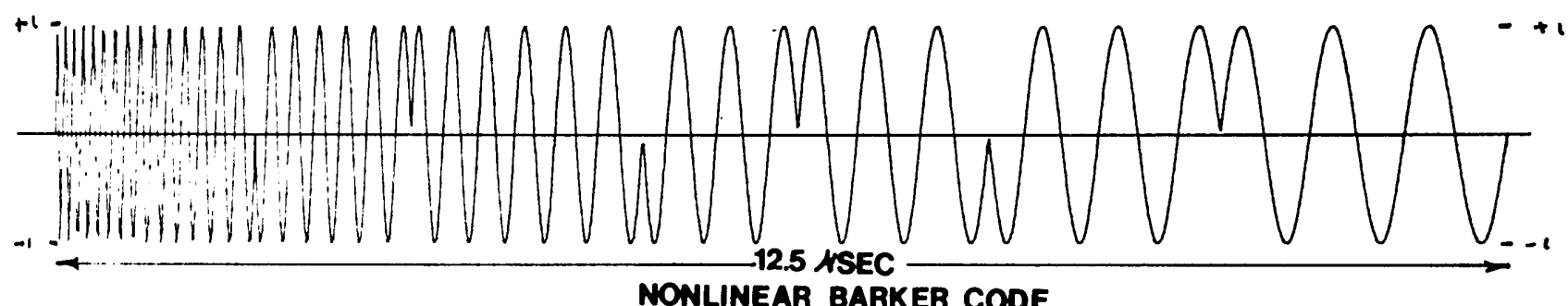

(b)

Fig. 4. Barker-coded biphase waveforms: (a) linear (normal) Barker; (b) nonlinear (coordinate transformed) Barker.

$\Delta \hat{t}$ and $B W$ determines $\Delta \hat{x}$, the waveform and system designers have separate and independent control of range and Doppler accuracy (a feature not available in most systems).

The relationships between $T$ and $T_{t}$, the durations of the reference and transmitted waveforms, respectively, are found from Eq. (5) to be

$$
T_{t}=\ln \left(T+t_{0}\right)-\ln \left(t_{0}\right)=\ln \left(1+T / t_{0}\right) .
$$

Thus, although $B W$ and $B W_{t}$ can be chosen independently, they are coupled by the corresponding time duration relationships. For example, if we attempt to increase $B W$ while keeping $T$ and $B W_{t}$ constant, the limit is set by the maximum $T_{t}$ that the system can accommodate. Thus $B W$ and $B W_{t}$ are not completely independent in practice, but are so within reasonable limits.

\section{Experimental Confirmation}

The full ambiguity surface for the space-variant processor of Fig. 2 using the nonlinear transmitted Barker-coded waveform of Eq. (6) was obtained using numerical analysis and computer simulation. (Optically produced output ambiguity surfaces and a comparison to the theoretical outputs will be published later.)

The 13-bit Barker-coded waveform of Eq. (3) [Fig. 4(a)] was nonlinearly coordinate transformed as in Eq. (6) with $t_{0}=T_{t}=2, \omega_{0}=200$, and $B W_{t}=50$ [Fig. 4(b)]. The ambiguity surface for the coordinate transformed version of this signal using Eq. (7) is shown in Fig. 5(a). A transmitted signal of larger $B W_{t}=130$ was then produced by changing the region of the coordinate transformation space used $\left(t_{0}=1\right)$. The resultant ambiguity surface is shown in Fig. 5(b).
From Fig. 5(b), we see that the ambiguity surface clearly exhibits a dominant peak as noted in Sec. IV with excellent sidelobe structure. The familiar Barker correlation pattern (six sidelobes of unit height on each side of a central peak 13 units high) appears along the $\hat{x}$ or Doppler axis now rather than along the $\tau$ (Range) as is convention. This occurs because the correlation is performed in Doppler, and time is incremented in the processors of Figs. 2 and 3. At the time $\hat{t}$ at which the target's range occurs, $P_{0}$ contains the normal Barker code of Eq. (3) displayed in $x$, whereas $P_{1}$ contains a matched filter of this normal Barker code. Hence, in $P_{2}$ we find the correlation of the Barker in $\hat{x}$ (or Doppler) as noted and shown in Fig. 5. As Doppler changes, the $P_{0}$ pattern (at the correct range or $\hat{t}$ ) simply shifts in $x$. (The $P_{2}$ pattern similarly shifts in $\hat{x}$.)

Comparing the ambiguity surfaces in Figs. 5(a) and 5 (b), we see that the larger $B W_{t}(130$ vs 50$)$ in Fig. 5(b) results in a sharper ambiguity peak in range $\hat{t}$ as predicted by theory. The higher sidelobe levels present in Fig. 5(a) correspond to the various $\hat{x}$ and $\hat{t}$ combinations that satisfy Eq. (13) for other distances $x$ in $P_{0}$. Computed ambiguity surfaces for other $B W_{t}, B W$, and $\omega_{0}$ values have been obtained; in all instances, the theoretical trends advanced earlier were verified.

The $\omega_{0}, B W, T, t_{0}$, etc. values used in the experiments are in relative units. To understand this, we recall that in the coordinate transformations $t=\exp x$ and $x=\ln t$, $t$ is time, and $x$ is space, but $\exp x$ and $\ln t$ should be dimensionless. Thus unit conversion parameters should be included in all expressions. Such rigorous formulations complicate understanding of the process and were thus not employed.

A final demonstration of the output ambiguity sur- 


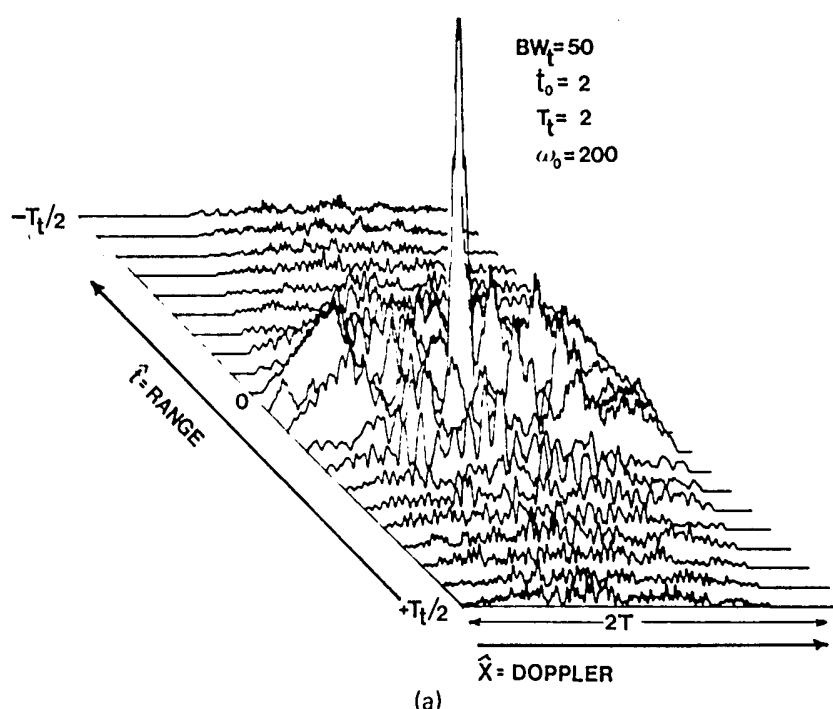

(a)

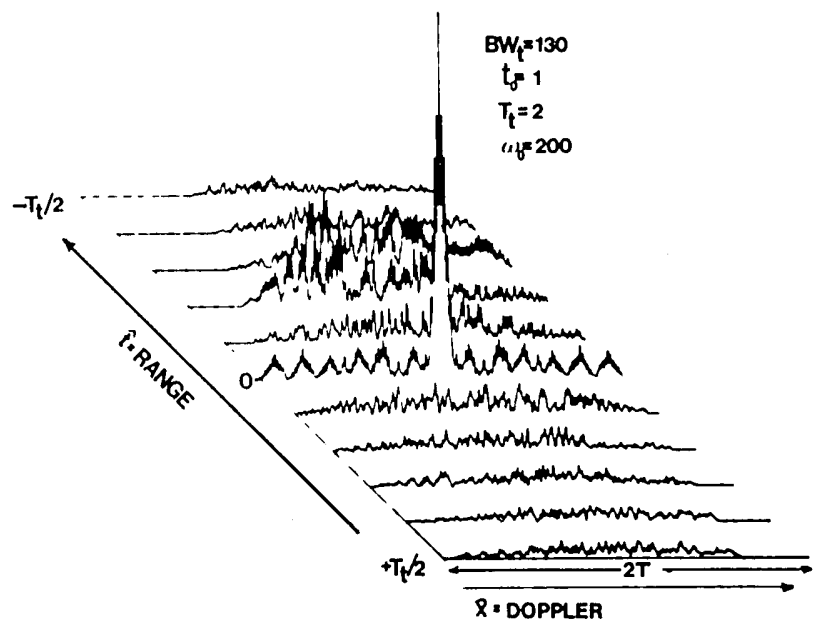

(b)

Fig. 5. Ambiguity surfaces for a nonlinear transmitted waveform with $\omega_{0}=200$ and $T_{t}=2$ : (a) $B W_{t}=50$ with $t_{0}=2$; (b) $B W_{t}=130$ with $t_{0}=1$.

face for a nonlinear waveform processed on a spacevariant system is shown in Fig. 6 . For this case, the parameters used in the Fig. 5(a) data were again used with two targets (at $0.125 T_{t}$ and $0.1 T_{t}$ ) introduced. The resultant plot shows the system's resolution in range as well as its ability to process multiple targets.

\section{Summary}

The use of nonlinear coded waveforms with spacevariant optical systems using coordinate transformation processing has been described. These waveforms were shown to result in nonlinear spread spectrum transmission with many novel properties. By proper use of the space and time variables, a single channel optical processor capable of producing the ambiguity surfaces for such waveforms is possible. Analysis of the resultant ambiguity surface has been shown to result in a valid ambiguity function. The waveform and system design flexibility afforded by such systems has been shown to be superior to those conventional waveforms

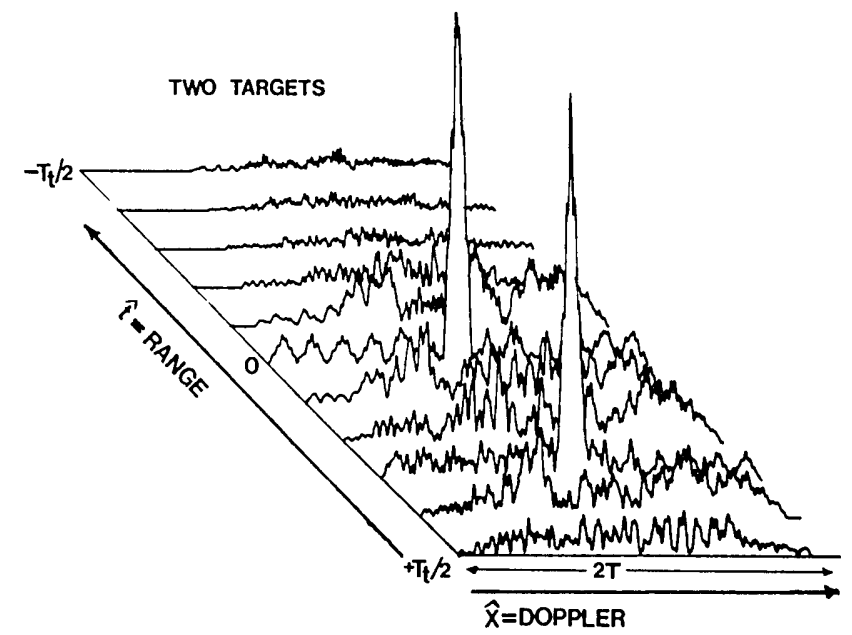

Fig. 6. Ambiguity surface for a nonlinear transmitted waveform showing the target resolution and multitarget capability of the system.

offer. Representative examples of the resultant ambiguity surfaces and the effects of varying waveform and coordinate transformation parameters have verified the theoretical remarks advanced.

The support of the National Science Foundation (grant ENG77-20038) for this work is gratefully acknowledged and appreciated.

\section{References}

1. D. Casasent and D. Psaltis, Appl. Opt. 15, 2015 (1976).

2. D. Casasent and M. Kraus, Opt. Commun. 19, 212 (1976).

3. R. Marks, J. Walkup, and M. Hagler, Proceedings International Optical Computer Conference (1976).

4. R. Marks et al., Appl. Opt. 16, 739 (1977).

5. P. Kellman and J. W. Goodman, Appl. Opt. 16, 2609 (1977).

6. M. Hagler et al., Proceedings International Optical Computers Conference (1978).

7. Special Issue on Optical Information Processing, Proc. Soc. Photo-Opt. Instrum. Eng. 83 (1976).

8. R. Bracewell, The Fourier Transforms and Its Applications (McGraw-Hill, New York, 1976).

9. G. M. Robbins and T. S. Huang, Proc. IEEE 60, 862 (1972).

10. A. Sawchuk, J. Opt. Soc. Am. 64, 138 (1974).

11. O. Bryngdahl, J. Opt. Soc. Am. 64, 1092 (1974).

12. D. Casasent and C. Szczutkowski, Opt. Commun. 19, 217 (1976).

13. D. Casasent and C. Szczutkowski, Proc. Soc. Photo. Opt. Instrum. Eng. 83, 91 (1976).

14. J. W. Goodman, P. Kellman, and E. W. Hansen, in Proceedings of International Optical Computer Conference (1976), p. 88.

15. J. W. Goodman, P. Kellman, and E. W. Hansen, Appl. Opt. 16, 733 (1977).

16. L. M. Deen, J. F. Walkup, and M. O. Hagler, Appl. Opt. 14, 2438 (1975).

17. T. Krile, R. Marks, J. Walkup, and M. Hagler, Appl. Opt. 16, 3131 (1977).

18. D. Psaltis and D. Casasent, Proceedings International Optical Computers Conference (1978).

19. D. Casasent and D. Psaltis, Opt Lett. 4, 18 (1979).

20. C. E. Cook and M. Bernfeld, Radar Signals (Academic, New York, 1967).

21. A. W. Rihaczek, Principles of High-Resolution Radar (McGraw-Hill, New York, 1969).

22. M. Born and E. Wolf, Principles of Optics (Pergamon, New York, 1965), p. 753 . 